\title{
Renal Transplantation in Antineutrophil Cytoplasmic Antibody- Associated Vasculitis: A Multicenter Experience
}

\author{
Duvuru Geetha ${ }^{1}$, Alfonso Eirin ${ }^{2}, K_{\text {Karin }}$ True $^{3}$, Maria Valentina Irazabal ${ }^{2}$, Ulrich Specks ${ }^{4}$, \\ Philip Seo ${ }^{5}$, Patrick Nachman ${ }^{3}$, and Fernando C. Fervenza ${ }^{2,6}$ \\ ${ }^{1}$ Division of Nephrology, Department of Medicine, Johns Hopkins University, Baltimore, MD \\ ${ }^{2}$ Division of Nephrology and Hypertension, Department of Medicine, Mayo Clinic College of \\ Medicine, Rochester, MN \\ ${ }^{3}$ Division of Nephrology and Hypertension, Department of Medicine, UNC Kidney Center, Chapel \\ Hill, NC \\ ${ }^{4}$ Division of Pulmonary and Critical Care, Department of Medicine, Mayo Clinic College of \\ Medicine, SW, Rochester, MN \\ ${ }^{5}$ Division of Rheumatology, Department of Medicine, Johns Hopkins University, Baltimore, MD
}

\section{Abstract}

Background-Antineutrophil cytoplasmic antibody (ANCA)-associated vasculitis (AAV) is a common cause of rapidly progressive glomerulonephritis resulting in end-stage renal disease (ESRD). The optimal timing of kidney transplantation (KTX) for ESRD as a result of AAV and the risk of AAV relapse after KTX are not well defined. We report our experience with AAV patients who underwent KTX at our institutions between 1996 and 2010. Median follow-up was 64 months.

Methods-Retrospective multicenter cohort study.

Results-Eighty-five patients ( 45 men/40 women; mean age 49 years) received a KTX for ESRD secondary to microscopic polyangiitis $(n=43)$ or Wegener's granulomatosis $(n=42)$.

Twenty-four patients underwent preemptive KTX and 69 received a living-donor KTX. All patients were in remission at the time of KTX. Fifty-eight patients received induction therapy. In 64 patients, maintenance immunosuppression was with prednisone, mycophenolate mofetil, and tacrolimus. At the time of KTX, 29 patients were ANCA-positive. The vasculitis relapse rate was 0.02 per patient-years and was not influenced by disease category, ANCA subtype, or remission duration before KTX. There were 23 rejection episodes in 13 patients with seven graft losses. Median serum creatinine at 1 year was $1.3 \mathrm{mg} / \mathrm{dL}$ in 75 patients with more than 1 year follow-up

\footnotetext{
Copyright (C) 2011 by Lippincott Williams \& Wilkins

${ }^{6}$ Address correspondence to: Fernando C. Fervenza, M.D., Ph.D., Division of Nephrology and Hypertension, Mayo Clinic College of Medicine, 200 First Street, SW, Rochester, MN 55905. fervenza.fernando@ mayo.edu.

The author's declare no conflict of interest.

D.G., U.S., and F.C.F. participated in research design, performance of research, and writing of the manuscript; A.E. and M.V.I. participated in data collection and analysis; and K.T., P.S., and P.N. participated in research design and performance of research.
} 
and $1.4 \mathrm{mg} / \mathrm{dL}$ at last follow-up. The graft and patient survival rates were $100 \%$ at 1 year, $97.9 \%$ and $93.4 \%$ at 5 years, and $79.0 \%$ and $67.4 \%$ at 10 years, respectively.

Conclusions-KTX is a safe and an effective option for treating ESRD secondary to AAV. Relapses are rare with current immunosuppression.

\section{Keywords}

ANCA vasculitis; Kidney transplantation; Immunosuppression; Outcomes

Pauci-immune crescentic glomerulonephritis remains the most common cause of rapidly progressive renal failure (1). The majority of cases are associated with the presence of circulating antineutrophil cytoplasmic antibodies (ANCA) (2). The two major subtypes of ANCA-associated vasculitis (AAV) are Wegener's granulomatosis (WG) and microscopic polyangiitis (MPA). Notwithstanding the advances in diagnosis and treatment of AAV, 20\% to $40 \%$ of patients developed end-stage renal disease (ESRD) (3-6). Kidney transplantation (KTX) has been shown to improve survival and quality of life among patients with ESRD, and several studies have demonstrated that KTX offers a survival benefit compared with maintenance dialysis $(7,8)$. Transplanted AAV patients also have lower vasculitis relapse rates compared with those who remain on dialysis $(6,9-12)$. A mean 10-year graft survival of $65.4 \%$ has been reported for patients with WG, which compares favorably with graft survival observed in other nonsystemic inflammatory conditions (13); however, as many as $50 \%$ of cases have been said to suffer a vasculitis relapse after KTX, and this may adversely affect allograft outcome $(3,4,14-21)$. A pooled analysis of 127 patients reported in 1999 indicated that AAV recurred in $17.3 \%$ of patients after KTX (22). The majority of these patients $(65 \%)$ received cyclosporine. In contrast, a much lower vasculitis relapse rate was observed in two single-center series published in recent years on AAV patients using modern immunosuppressive agents $(23,24)$. Gera et al. (23), in a single-center series of 35 transplant recipients with diagnoses of AAV reported nonrenal relapses in three patients $(8.6 \%)$. No clear risk factor to relapse emerged and no detrimental effect to renal function was found. In addition, another single-center analysis of 17 patients with AAV who underwent KTX identified relapses in three patients over a median follow-up of 37 months (24). More recently, Little et al. (25) reported relapses in only 5 of 107 transplanted AAV patients (4.7\%). Overall graft survival was $70 \%$ after 10 years. ANCA status per se was not significantly associated with graft failure, and the strongest predictor of death was transplantation less than 1 year from the time of vasculitis remission (25). This study included patients transplanted over a 20-year period and did not have details of posttransplant immunosuppression.

These discrepancies and the paucity of information regarding long-term transplant outcomes for AAV in the modern era of immunosuppression led us to extend our previous observations by conducting this multicenter study to address questions regarding the influence of waiting period after remission is achieved before KTX, influence of disease subtypes, ANCA status at the time of KTX, and the effect antirejection regimen on graft outcome and vasculitis relapse rate in this patient population. 


\section{RESULTS}

\section{Patient Characteristics}

A total of 85 patients received KTX for ESRD secondary to AAV. Forty-two patients had WG and 43 patients had MPA. The clinical characteristics of the cohort are listed in Table 1. The mean age at transplantation was $49 \pm 18.2$ years. Sixty-nine patients received a KTX from a living-related donor. Twenty-four patients were transplanted preemptively. The remaining 61 patients spent a median of 17.5 (range, 1-120) months on dialysis before receiving a KTX. All patients were in clinical remission at the time of KTX, with 57 of 85 patients $(67.0 \%)$ in remission for more than 12 months. At the time of transplantation, ANCA testing by immunofluorescence was carried out in 75 of the 85 patients and was positive in 29 of the 75 patients $(38.7 \%)$.

\section{Transplant Immunosuppression}

Fifty-eight patients received induction therapy with the majority of them receiving antithymocyte globulin (Table 2). Sixty-four patients (75\% of total) received maintenance immunosuppression with prednisone, mycophenolate mofetil, and tacrolimus. There were no differences in the dose of mycophenolate mofetil used or target tacrolimus drug levels in patients who were ANCA positive compared with patients who were ANCA negative at the time of transplant. Eighty-one patients were maintained on steroids and the remaining four were on a steroid avoidance protocol.

\section{Transplant Outcomes}

Clinical outcomes were followed-up until patient death, graft loss with return to dialysis, or most recent documentation of graft status. The median post-KTX follow-up was 64 (range, 3-165) months. There were 23 biopsy-proven rejection episodes in 13 patients. Six patients developed biopsy-proven polyomavirus (BK)-associated nephropathy. The median serum creatinine at 1 year was $1.3 \mathrm{mg} / \mathrm{dL}$ in the 75 patients who had more than 1 year of follow-up. The median serum creatinine at last follow-up was $1.4 \mathrm{mg} / \mathrm{dL}$. There was no difference in the median serum creatinine at the last follow-up between patients with WG and MPA (1.4 vs. 1.3, $P=0.09)$. Similarly, there was no difference in median serum creatinine at the last follow-up of patients who were ANCA positive at the time of transplant compared with those who were ANCA negative (1.4 vs. 1.3, $P=0.31$ ). There were seven graft losses in this cohort. In three patients, graft loss was due to noncompliance with immunosuppression, three patients developed chronic allograft nephropathy and one patient developed renal cell carcinoma in the KTX.

Kaplan-Meier plots for overall graft survival and death-censored graft survival are presented in Figure 1. As shown, a total of 11 patient deaths occurred between 25 and 152 months after KTX. Eight patients had functioning KTX at the time of death, whereas three patients lost KTX before their death. An additional four grafts were lost between 70 and 84 months post-KTX. Deaths were due to cancer $(n=3)$, sepsis $(n=1)$, amyloidosis $(n=1)$, intracranial hemorrhage $(\mathrm{n}=2)$, and unexplained sudden death $(\mathrm{n}=4)$. Overall graft survival at 5 years was $97.9 \%$ with death-censored graft survival of $93.4 \%$ at this time point. 


\section{Vasculitis Outcomes and Risk of Relapse}

At the time of transplant, 75 patients had been tested for ANCA. Twenty-nine (38.7\%) patients were ANCA positive and the rest were ANCA negative. A total of eight relapses occurred in seven patients ( 5 with WG and 2 with MPA; Table 3). The vasculitis relapse rate was 0.02 per patient year. All flares occurred in the presence of detectable circulating ANCA. Two patients with MPA, one ANCA positive and one unknown, and both patients in remission for more than 1 year before KTX developed a necrotizing glomerulonephritis within 3 months of KTX. One patient responded to a 3-month course of cyclophosphamide, and another patient responded to a change in immunosuppression from azathioprine to mycophenolate mofetil. One patient with WG developed two episodes of a pulmonary-renal syndrome 45 and 55 months after KTX; the latter occurred in the setting of non-compliance with his immunosuppression and resulted in graft loss. A second patient with WG developed lung hemorrhage followed by pneumonia and methicillin resistant staphylococcus aureus (MRSA) sepsis 44 months after KTX; this patient died of sepsis 2 months later. The symptomatic presentation of the remaining cases of relapse in WG involved upper respiratory tract alone $(n=1)$; multiple joints $(n=1)$; and upper respiratory tract, joints, and skin $(n=1)$. None of the remaining patients experienced renal or extra renal relapse of vasculitis after KTX after a median observation period of 64 months. Four of the seven patients who experienced a relapse were ANCA positive at the time of KTX, one patient was ANCA negative, and in two patients, the ANCA status at the time of KTX was unknown. We found that being ANCA positive at the time of the KTX was associated with an increased odds of developing a vasculitis relapse (95\% confidence interval 0.998 144.932, $P=0.05$ ). However, this finding is limited by the fact that in two patients their ANCA status was unknown and could not be included in the analysis. There was no correlation between relapse and ANCA type (p-ANCA vs. c-ANCA $P=0.7$ ). There was no statistically significant difference regarding the time on dialysis before KTX between relapsers and nonrelapsers. There was no significant difference in relapse rate between KTX patients who had been in remission for less than 12 months and those who had been in remission for a longer period. Although patients with WG seemed to have a higher relapse rate $(11.9 \%)$ when compared with patients with MPA (4.7\%), this difference did not reach statistical significance $(P=0.6)$. There was also no difference in relapse rate between the three centers. There was no significant difference in use of induction therapy $(P=0.4)$ and use of mycophenolate mofetil $(P=0.7)$ in patients who experienced a relapse versus patients who did not experience a relapse.

\section{DISCUSSION}

This multicenter study reports allograft outcomes and vasculitis relapse rates in $85 \mathrm{AAV}$ patients who underwent KTX in the era of modern immunosuppression. Of the 85 patients reported in this study, 52 have been reported in earlier studies as single-center experiences $(23,24)$. We have now extended this observation to an additional 33 patients and reported outcomes with a longer follow-up on the 52 patients reported in our earlier studies. This study represents the largest and most uniformed series of AAV patients who underwent KTX in the era of modern immunosuppression, and it is the one with the longest follow-up. We sought to address questions regarding the influence of remission time before the KTX, 
influence of disease subtypes and ANCA status at the time of KTX on vasculitis relapse, the effect anti-rejection regimen on graft outcome, and vasculitis relapse rate and the safety of immunosuppression in this patient population. Overall graft survival was 120 months. There was no significant difference in vasculitis relapse rate among patients who were transplanted less than 3 months of entering into clinical remission compared with those who remained in remission longer $(P=0.2)$. We found that ANCA positivity at the time of transplantation tended to be associated with an increased risk for a vasculitis relapse, but these data need to be taken with caution in view of the small number of patients, the fact that in two patients, ANCA status at the time of KTX was unknown, and in one patient, the relapse occurred in the setting of noncompliance with medication. More important, ANCA positivity per se at the time of transplantation was not associated with adverse graft survival. There was no difference in median serum creatinine at the last follow-up between different disease categories or ANCA subtype.

Our results confirm earlier reports demonstrating that KTX is an excellent option for the treatment of patients with ESRD secondary to AAV with lower death rate and lower vasculitis relapse rates compared with AAV patients on dialysis. Lionaki et al. (26) reported 59 deaths among 93 AAV patients on dialysis over a median follow-up time of 63 months. The corresponding mortality rate was 0.31 deaths per person per year. Data from the Collaborative Transplant Study show that patients with AAV who received a deceased donor KTX had a 10-year patient survival rate of $80 \%$ (13). Similarly, in a case series of 22 AAV patients who underwent KTX, Allen et al. (3) reported post-KTX patient survival of $100 \%$ and $85 \%$ at 1 and 5 years, respectively, with graft survival of $86 \%$ and $69 \%$, respectively. In our previous single-center analysis of KTX in patients with AAV using modern immunosuppressive agents, the overall and death-censored graft survivals were $94 \%$ and $100 \%$, respectively, 5 years post-transplantation (23). The overall and death-censored graft survival at 5 years in this cohort was $93.4 \%$ and $97.9 \%$, respectively.

Transplanted AAV patients experience lower vasculitis relapse rate compared with AAV patients on dialysis and AAV patients with chronic kidney disease who are not on dialysis. Allen et al. (3) reported a vasculitis relapse rate of 0.09 relapses per patient per year in 37 patients with AAV on dialysis compared with a vasculitis relapse rate of 0.02 per patient per year in 22 transplanted AAV patients. Lionaki et al. (26) reported a vasculitis relapse rate of 0.08 episodes per person year in 93 patients with AAV on dialysis and a vasculitis relapse rate of 0.16 episodes per person year in 359 patients in AAV patients with renal involvement who had not reached ESRD. There has been, however, great variability regarding relapse rates in AAV patients after $\operatorname{KTX}(3,14-20)$. A pooled analysis by Nachman et al. (22), reported an overall relapse rate of $17 \%$, with an average time from KTX to relapse of 31 months. On the other hand, Schmitt et al. (19) reported a vasculitis relapse rate of 0.1 episodes per patient per year in 20 patients with WG with a mean follow-up of 48 months. Similarly, Haubitz et al. (16) reported a vasculitis relapse rate of 0.06 per patient per year. In a report of nine patients with AAV and KTX, the vasculitis relapse rate was 0.04 per patient per year (27). The reports mentioned above were based on data from patients mainly treated with prednisone, cyclosporine, and azathioprine for maintenance immunosuppression. More recently, however, a relapse rate of 0.005 per patient per year was reported in 17 patients 
with AAV whose maintenance KTX immunosuppression was based on the use of tacrolimus and mycophenolate mofetil (24). Moreover, Gera et al. (23) reported a relapse rate of 0.02 per patient per year in $35 \mathrm{AAV}$ patients undergoing KTX using modern immunosuppressive agents. Our data confirm and strengthen prior observations that, with current immunosuppression therapy, vasculitis relapse rate after KTX is low and does not affect graft survival.

Because of the risk of recurrent disease in the setting of active disease, it is generally recommended to wait until clinical remission is achieved before proceeding to KTX. The optimal duration of clinical remission required before transplantation is not clear. Little et al. showed that KTX shortly after induction of remission was the strongest predictor of mortality after transplantation. However, in our study, the vasculitis relapse rate was not influenced by duration of remission. In contrast to anti-glomerular basement membrane (GBM) disease where persisting anti-GBM antibodies are associated with a higher recurrence rate, evidence is accumulating that the presence of positive ANCA should not preclude transplantation in patients who are clinically in remission. C-ANCA was detectable in 8 of 12 WG patients at the time of transplantation in the study by Schmitt et al. (19) and in seven of the eight patients in a case series by Rostaing et al. (18). Only one of the patients in the latter group relapsed; four others remained ANCA positive during follow-up but remained in remission. Similarly, Deegens et al. (28) studied the outcome of KTX in 33 patients with AAV transplanted between 1968 and 2000 under varying immunosuppressive regimens. ANCA testing was carried out in 18 patients around the time of transplantation, and positive ANCA by immunofluorescence was found in eight patients ( 8 of the $18 ; 44 \%$ ). Only one patient (3\%) with WG developed an extra renal relapse, with none of the patients developing a renal relapse. In a pooled analysis of recurrent AAV after renal transplant in 33 patients, there was no significant difference in the relapse rate between those with or without detectable ANCA titers at the time of transplantation (22). There was also no difference in the rate of relapse after KTX based on ANCA pattern or antigen specificity. In our previous study (23), there was a trend toward higher relapse rate among patients who had a positive ANCA test before KTX (13\% vs. 5\%) but the difference was not statistically significant and relapses among both groups were infrequent and clinically manageable. A similarly trend was observed in the current study (13.8\% vs. $2.2 \%$ ), but again this needs to be taken with caution because of the reason discussed above. Thus, ANCA status at the time of transplant should not preclude KTX.

Differences in renal outcomes and risk of relapse have been reported between patients with MPA versus WG. In a single-center case series, the median serum creatinine at the time of last follow-up was higher among patients with WG than MPA (1.3 vs. $1.0 \mathrm{mg} / \mathrm{dL}$ ) (24). In contrast to this, we did not find a difference in median serum creatinine at last follow-up when patients with WG were compared with MPA (1.2 vs. $1.3 \mathrm{mg} / \mathrm{dL}, P=0.09)$.

The present cohort represents the largest experience with KTX for AAV and is specifically relevant to current practice trends in KTX. The study is subject to the limitations inherent to a retrospective analysis. The data were pooled from three centers. However, there were no significant differences in immunosuppressive drug use, allograft function, or vasculitis relapse rates between the three centers. Vasculitis activity at the time of KTX was defined 
based on clinical criteria and did not use standardized Birmingham Vasculitis Assessment Score (WG). Nonetheless, our results showed that with current immunosuppression the risk of recurrence of vasculitis post-KTX is low and supports the conclusion that KTX is the treatment of choice for patients with ESRD due to AAV who are in clinical remission at the time of transplantation regardless of ANCA status.

\section{MATERIALS AND METHODS}

\section{Study Population}

The study population included all KTX recipients from 1996 to 2010 who had AAV listed as the primary cause of ESRD and were followed-up at Mayo Clinic, Rochester, Minnesota; Johns Hopkins Hospital, Baltimore, Maryland; and University of Chapel Hill, North Carolina. The study protocol was approved by the institutional review board at each institution. For the purposes of this study, the diagnosis of systemic vasculitis required documentation of the following: (a) a diagnosis of vasculitis proven by biopsy and (b) positive ANCA testing. Patients were classified as WG or MPA as defined by the Chapel Hill Consensus Conference $(2,29)$.

\section{Data Acquisition}

Clinical, laboratory, and histologic data were abstracted from review of medical records of all cases meeting study entry criteria. Data regarding patient demographics, donor source, prior KTX, disease subtype, ANCA type and status at time of transplant, duration of dialysis pretransplant when applicable, duration of remission before transplant, presence of delayed graft function, data on induction therapy, maintenance immunosuppressive regimen, serum creatinine at 1 year and last follow-up, episodes of allograft rejection and details of treatment of allograft rejection, occurrence of vasculitis relapse posttransplant, relapse type and treatment of relapse, and occurrence of graft loss. Overall and death-censored graft survival rates were examined by Kaplan-Meier survival curves. Data are presented as means ( \pm standard deviation) or medians (range) when appropriated. All statistics were performed using JMP software, version 8.0 (SAS Institute Inc., Cary, NC).

\section{References}

1. Jennette JC. Rapidly progressive crescentic glomerulonephritis. Kidney Int. 2003; 63:1164. [PubMed: 12631105]

2. Jennette JC, Falk RJ, Andrassy K, et al. Nomenclature of systemic vasculitides. Proposal of an international consensus conference. Arthritis Rheum. 1994; 37:187. [PubMed: 8129773]

3. Allen A, Pusey C, Gaskin G. Outcome of renal replacement therapy in antineutrophil cytoplasmic antibody-associated systemic vasculitis. J Am Soc Nephrol. 1998; 9:1258. [PubMed: 9644636]

4. Cohen BA, Clark WF. Pauci-immune renal vasculitis: Natural history, prognostic factors, and impact of therapy. Am J Kidney Dis. 2000; 36:914. [PubMed: 11054347]

5. Westman KW, Bygren PG, Olsson H, et al. Relapse rate, renal survival, and cancer morbidity in patients with Wegener's granulomatosis or microscopic polyangiitis with renal involvement. J Am Soc Nephrol. 1998; 9:842. [PubMed: 9596082]

6. Schmitt WH, van der Woude FJ. Organ transplantation in the vasculitides. Curr Opin Rheumatol. 2003; 15:22. [PubMed: 12496506] 
7. Schnuelle P, Lorenz D, Trede M, et al. Impact of renal cadaveric transplantation on survival in endstage renal failure: Evidence for reduced mortality risk compared with hemodialysis during longterm follow-up. J Am Soc Nephrol. 1998; 9:2135. [PubMed: 9808102]

8. Wolfe RA, Ashby VB, Milford EL, et al. Comparison of mortality in all patients on dialysis, patients on dialysis awaiting transplantation, and recipients of a first cadaveric transplant. N Engl J Med. 1999; 341:1725. [PubMed: 10580071]

9. Fauci AS, Balow JE, Brown R, et al. Successful renal transplantation in Wegener's granulomatosis. Am J Med. 1976; 60:437. [PubMed: 769547]

10. Lyons GW, Lindsay WG. Renal transplantation in a patient with Wegener's granulomatosis. Am J Surg. 1972; 124:104. [PubMed: 4557649]

11. Moises J, Torregrosa JV, Ybarra J, et al. Renal transplantation in a C-ANCA(+) patient with Behcet disease and rapidly progressive glomerulonephritis. Clin Nephrol. 2004; 61:357. [PubMed: 15182132]

12. Besbas N, Ozaltin F, Tinaztepe K, et al. Successful renal transplantation in a child with ANCAassociated microscopic polyangiitis. Pediatr Nephrol. 2003; 18:696. [PubMed: 12743792]

13. Schmitt WH, Opelz G, Van Der Woude FJ. Renal transplantation is safe and successful in Wegener's granulomatosis: Data from the Colloborative Transplant Study. J Am Soc Nephrol. 2002; 13:564A.

14. Frasca GM, Neri L, Martello M, et al. Renal transplantation in patients with microscopic polyarteritis and antimyeloperoxidase antibodies: Report of three cases. Nephron. 1996; 72:82. [PubMed: 8903866]

15. Grotz W, Wanner C, Rother E, et al. Clinical course of patients with antineutrophil cytoplasm antibody positive vasculitis after kidney transplantation. Nephron. 1995; 69:234. [PubMed: 7753254]

16. Haubitz M, Kliem V, Koch KM, et al. Renal transplantation for patients with autoimmune diseases: Single-center experience with 42 patients. Transplantation. 1997; 63:1251. [PubMed: 9158017]

17. Kuross S, Davin T, Kjellstrand CM. Wegener's granulomatosis with severe renal failure: Clinical course and results of dialysis and transplantation. Clin Nephrol. 1981; 16:172. [PubMed: 7028336]

18. Rostaing L, Modesto A, Oksman F, et al. Outcome of patients with antineutrophil cytoplasmic autoantibody-associated vasculitis following cadaveric kidney transplantation. Am J Kidney Dis. 1997; 29:96. [PubMed: 9002536]

19. Schmitt WH, Haubitz M, Mistry N, et al. Renal transplantation in Wegener's granulomatosis. Lancet. 1993; 342:860. [PubMed: 8104282]

20. Wrenger E, Pirsch JD, Cangro CB, et al. Single-center experience with renal transplantation in patients with Wegener's granulomatosis. Transpl Int. 1997; 10:152. [PubMed: 9090004]

21. Clarke AE, Bitton A, Eappen R, et al. Treatment of Wegener's granulomatosis after renal transplantation: Is cyclosporine the preferred treatment? Transplantation. 1990; 50:1047. [PubMed: 2256145]

22. Nachman PH, Segelmark M, Westman K, et al. Recurrent ANCA-associated small vessel vasculitis after transplantation: A pooled analysis. Kidney Int. 1999; 56:1544. [PubMed: 10504506]

23. Gera M, Griffin MD, Specks U, et al. Recurrence of ANCA-associated vasculitis following renal transplantation in the modern era of immunosupression. Kidney Int. 2007; 71:1296. [PubMed: 17410100]

24. Geetha D, Haas M, Kraus ES, et al. Renal transplant in Wegener's granulomatosis compared to microscopic polyangiitis. J Rheumatol. 2010; 37:1705. [PubMed: 20516028]

25. Little MA, Hassan B, Jacques S, et al. Renal transplantation in systemic vasculitis: When is it safe? Nephrol Dial Transplant. 2009; 24:3219. [PubMed: 19602476]

26. Lionaki S, Hogan SL, Jennette CE, et al. The clinical course of ANCA small-vessel vasculitis on chronic dialysis. Kidney Int. 2009; 76:644. [PubMed: 19536079]

27. Elmedhem A, Adu D, Savage CO. Relapse rate and outcome of ANCA-associated small vessel vasculitis after transplantation. Nephrol Dial Transplant. 2003; 18:1001. [PubMed: 12686678] 
28. Deegens JK, Artz MA, Hoitsma AJ, et al. Outcome of renal transplantation in patients with pauciimmune small vessel vasculitis or anti-GBM disease. Clin Nephrol. 2003; 59:1. [PubMed: 12572924]

29. Leavitt RY, Fauci AS, Bloch DA, et al. The American College of Rheumatology 1990 criteria for the classification of Wegener's granulomatosis. Arthritis Rheum. 1990; 33:1101. [PubMed: 2202308] 


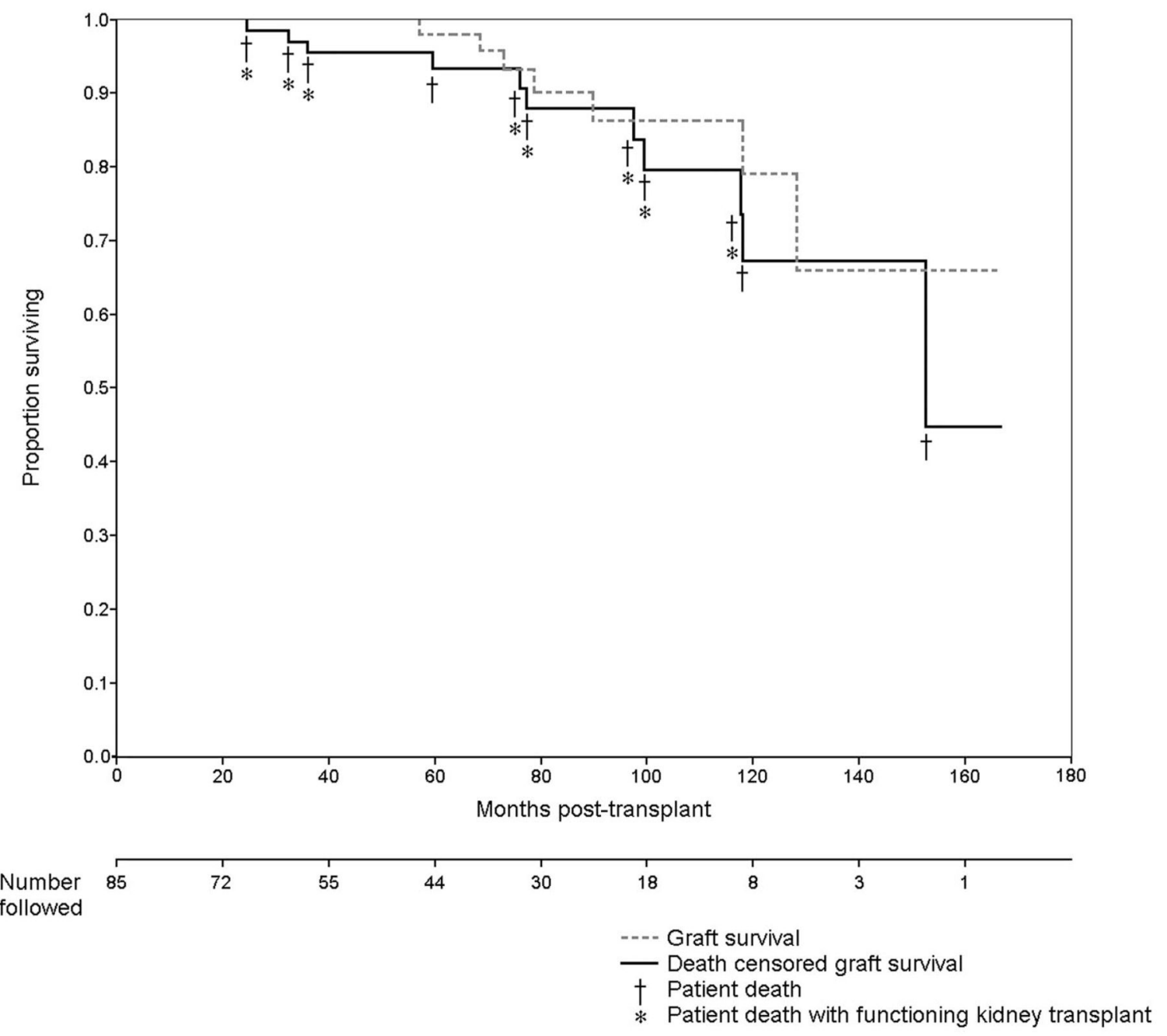

FIGURE 1.

Kaplan-Meier curves are shown for overall kidney transplant survival (graft survival) and death-censored kidney transplant survival among 85 recipients with ESRD due to AAV. ESRD, end-stage renal disease; AAV, antineutrophil cytoplasmic antibody-associated vasculitis. 


\section{TABLE 1}

Baseline characteristics of KTX recipients with native kidney failure due to AAV

$\begin{array}{lc}\text { Patient number } & 85 \\ \text { Form of AAV (WG:MPA) } & 42: 43 \\ \text { History of prior KTX, n (\%) } & 8(9.4) \\ \text { ANCA-positive at time of KTX, n (\%) } & 29 \text { of } 75(38.7) \\ \text { Age at transplant (mean } \pm \text { SD, yr) } & 49 \pm 18.2 \\ \text { Gender (M:F) } & 45: 40 \\ \text { Donor source, n (\%) } & 54(63.5) \\ \quad \text { LRD } & 15(17.6) \\ \quad \text { LURD } & 16(18.8) \\ \quad \text { DD } & 24(28.2) \\ \text { Preemptive KTX, n (\%) } & 17.5(1-120) \\ \text { Time on dialysis, median (range), mo } & 12(4-120) \\ \text { Time between last AAV activity and KTX, median (range), mo } & \end{array}$

ANCA, antineutrophil cytoplasmic antibody; AAV, ANCA-associated vasculitis; DD, deceased donor; LRD, living-related donor; LURD, livingunrelated donor; MPA, microscopic polyangiitis; WG, Wegener's granulomatosis; KTX, kidney transplantation; SD, standard deviation. 


\section{TABLE 2}

Kidney transplant-related immunosuppression and posttransplant outcomes

\begin{tabular}{lc}
\hline & $\mathbf{n}(\%)$ \\
\hline Induction immunosuppression & $27(31.8)$ \\
None & $14(16.5)$ \\
IL-2 receptor antagonist & $41(48.2)$ \\
Antithymocyte globulin & $3(3.5)$ \\
Anti-CD52 (alemtuzumab) & \\
Primary oral immunosuppressant & $64(75.0)$ \\
TAC/MMF/P & $7(8.2)$ \\
CsA/MMF/P & $2(2.4)$ \\
CsA/SRL/P & $2(2.4)$ \\
SRL/MMF/P & $2(2.4)$ \\
TAC/AZA/P & $1(1.2)$ \\
TAC/SRL/P & $4(4.7)$ \\
TAC/MMF & $2(2.4)$ \\
TAC/P & $1(1.2)$ \\
CsA/P & $5.4(3.4)$ \\
Follow-up (mean $\pm S D$, yr) & $13(15.3)$ \\
Acute rejection, n (\%) & $6(7.1)$ \\
Polyomavirus (BK)-associated nephropathy & $1.3(0.8-2.4)$ \\
SCr at 1 yr post-KTX, median (range), mg/dL & \\
Clinical relapse of AAV & $7 / 85(8.2)$ \\
Total group & $5 / 42(11.9)$ \\
WG group & $2 / 39(5.1)$ \\
MPA group & $4 / 29(13.8)$ \\
ANCA-positive at time of KTX & $1 / 46(2.2)$ \\
ANCA-negative at time of KTX & $2 / 10(20.0)$ \\
ANCA-unknown at time of KTX & \\
\hline
\end{tabular}

AZA, azathioprine; CsA, cyclosporine; MMF, mycophenolate mofetil; MPA, microscopic polyangiitis; SRL, sirolimus; TAC, tacrolimus; WG, Wegener's granulomatosis; ANCA, antineutrophil cytoplasmic antibody; KTX, kidney transplantation; IL, interleukin; AAV, ANCA-associated vasculitis. 


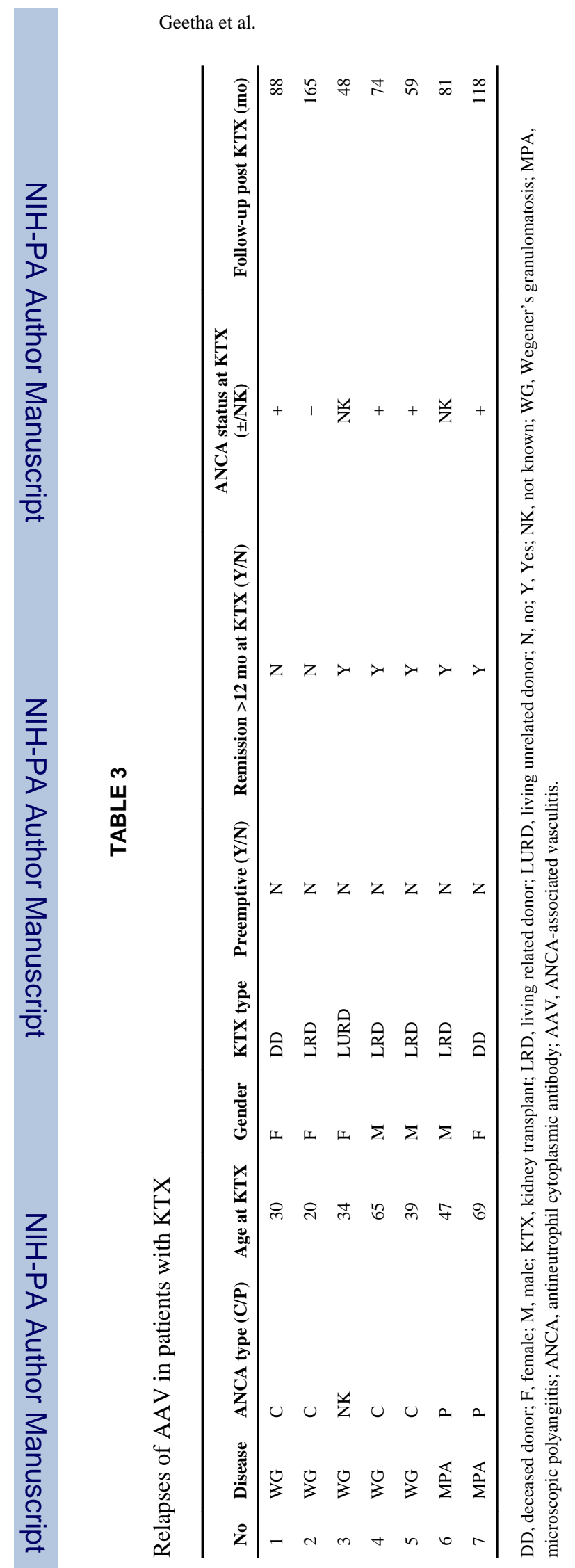

Transplantation. Author manuscript; available in PMC 2014 July 15. 